LAWRENCE LIVERMORE N A T IO N A L LABORATORY
Laser Heated High Density Fluids Probed by Coherent Anti-Stokes Raman Spectroscopy

B. J. Baer, C.-S. Yoo

May 25, 2004

Review of Scientific Instruments 
This document was prepared as an account of work sponsored by an agency of the United States Government. Neither the United States Government nor the University of California nor any of their employees, makes any warranty, express or implied, or assumes any legal liability or responsibility for the accuracy, completeness, or usefulness of any information, apparatus, product, or process disclosed, or represents that its use would not infringe privately owned rights. Reference herein to any specific commercial product, process, or service by trade name, trademark, manufacturer, or otherwise, does not necessarily constitute or imply its endorsement, recommendation, or favoring by the United States Government or the University of California. The views and opinions of authors expressed herein do not necessarily state or reflect those of the United States Government or the University of California, and shall not be used for advertising or product endorsement purposes. 


\title{
Laser heated high density fluids probed by Coherent Anti-Stokes Raman Spectroscopy
}

\section{Bruce J. Baer and Choong-Shik Yoo}

H-Division, Lawrence Livermore National Laboratory, University of California, Livermore, CA 94550

\begin{abstract}
We describe an integrated experimental method of Coherent Antistoke Raman Spectroscopy (CARS), laser-heating and diamond-anvil cell (DAC) technologies probing molecular vibrations of transparent molecular fluids at the pressure-temperature conditions of energetic detonation and Giant planetary interiors. In this method, we use a microfabricated metal toroid to conductively heat a surrounding transparent sample in a DAC, using a $\mathrm{CW} \mathrm{Nd}$ :YLF laser. The laser is operated at a $\mathrm{TEM}_{01} *$ mode to match the shape of the toroid and thus produces a uniform heating area. The CARS probe utilizes two pulsed lasers with similar cavity lengths: a commercial narrow-band mode-locked Q-switched $\mathrm{Nd}$ :YAG laser and a home-built broadband dye laser. A strong CARS signal is then produced from the sample in the central region of laser-heated metal toroid where the two laser pulses spatially and temporally coincide. In this paper, we will demonstrate that this technique is capable of producing high quality vibrational spectra from nitrogen fluid above $2000 \mathrm{~K}$ and $13 \mathrm{GPa}$, where the application of spontaneous Raman spectroscopy is limited because of intense thermal radiation.
\end{abstract}




\section{INTRODUCTION}

Spectroscopic measurements of materials under the extreme conditions of high pressure and high temperature are rare. Studies of explosive detonations, planetary interiors and novel material properties require information about the structure, thermodynamics, energy transfer, and chemical reactivity of high density fluids at these extremes. Such data is necessary to adequately understand and model the molecular potentials even of simple molecular systems. Many experiments at ambient temperature typically probe only the ground state, but at sufficiently elevated temperatures a significant fraction of molecules are in a vibrationally excited state. Furthermore, the effect of

pressure induced molecular dissociation can be addressed by observing the changes in the vibrational potential with increasing compression. A challenge to obtain such data is the lack of a proper spectroscopic probe at these formidable high pressure-temperature conditions.

A handful of studies that obtained the vibrational spectra of simple molecules such as $\mathrm{N}_{2}, \mathrm{O}_{2}$, or $\mathrm{CO}$ at high temperatures $(>2000 \mathrm{~K})$ and pressures $(>10 \mathrm{GPa})$ have been performed under shock conditions for both the pure simple molecular systems [1-3] and their mixtures. [4-7] However, there have been no similar measurements to date under static high pressure-temperature conditions. The difficulty of obtaining spectra under shock conditions is due to the brevity of time under which the sample is compressed and its somewhat restricted geometry. For the diamond-anvil cell, unlike the shock experiments, achieving a uniform high temperature is the greatest obstacle. In either experiment, the large thermal background must be overcome so that a satisfactory vibrational spectrum can be obtained.

In this paper we focus on using $\mathrm{N}_{2}$ as a test material. The highest temperature previously reported temperature for which vibrational spectra of high-density fluid $\mathrm{N}_{2}$ in a diamond-anvil cell has been obtained is about 900K. [8] Most likely, this is the highest temperature one can ever hope to achieve by external heating methods on a sample of 
nitrogen in a DAC due to fluid nitrogen difffusion through the gasket diamond interface. As difficult as this technical achievement was to accomplish, much higher temperatures are needed if molecular potentials are to be determined. Also, this static experiment is in dramatic comparison to the highest temperature for which vibrational spectra of nitrogen in a shock experiment has been obtained, 5400K. [1]

To achieve high temperatures $(>2000 \mathrm{~K})$ in a diamond-anvil cell (DAC) laser heating must be used. However, most materials are not likely to electronically or vibrationally couple to the available laser light. Homodiatomic molecules have no dipole moment and their electronic transitions are typically in the ultraviolet, where the diamonds are opaque. Also, the laser light absorbed must rapidly end up as a significant quantity of heat and not be radiated out as luminescence or bleach the sample. Therefore, an indirect heating method must be used to ensure that this technique is useful for the widest possible range of analytes. One of the most critical requirements for laser heating is that uniform temperatures be produced within a sample. While this occurs automatically behind the shock front of a gas gun experiment, a laser heated sample in a diamond-anvil cell often has an extremely high temperature gradient since the diamonds, the best known thermal conductor, must remain relatively close to ambient temperature.

\section{EXPERIMENTAL PROCEDURE}

Typically, a DAC sample is held inside the aperture of a metal gasket and compressed between the culets of two opposing modified brilliant cut anvils. Our samples use Rhenium as the gasket material in order to achieve the thickest possible sample. There are, however, reports of other gasket preparations that result in a thicker sample. [9] To heat this sample, a metal toroid is placed inside the gasket aperture before sample loading. Gasket apertures are 
produced to be only slightly larger than this toroid so that the toroid will be held in place, but in poor thermal contact with the gasket once the sample is loaded at pressure.

The toroid target must be either chemically inert with the analyte at high temperature or form a compound that acts as a protective layer between toroid and sample. Furthermore, the toroid must withstand extremely high temperatures (i.e.: not melt), and absorb some of the laser light we wish to use. Typically, tungsten, tantalum and platinum are tested to see which works best. For nitrogen, we found that tungsten was the best choice.

Metal toroids are fabricated using electric discharge machining (EDM) by the following method. A thin foil $(\leq 25 \mu \mathrm{m})$ of metal $(\mathrm{W}, \mathrm{Ta}$ or $\mathrm{Pt})$ is epoxied onto a small stainless steel square $\left(\sim 1 \mathrm{~cm}^{2}\right)$ using a thin $(\leq 5 \mu \mathrm{m})$ layer of conducting epoxy. A small piece $(\sim 3 / 8$ " in length) of hypodermic tubing is used as an EDM bit to drill out a circular trench down to the epoxy or, preferably, the stainless steel backing. The tubing has a small internal diameter, usually less than 100 microns. The small disk inside the trench is drilled down the center (also by EDM) using a small $(<25 \mu \mathrm{m})$ diameter wire producing a toroid. The thickness of the toroid can be reduced further using EDM with a drill bit slightly larger than toroid outer diameter. Our toroids typically have a $90 \mu \mathrm{m}$ outer diameter, a $25 \mu \mathrm{m}$ inner diameter, and are $15 \mu \mathrm{m}$ thick. The toroid is thoroughly cleaned and then placed inside the gasket aperture so that it will not touch the diamond surface where the heating laser beam will enter. It is best to have at least 10 microns spacing between the culet and diamond. Figure 1 shows this configuration of the DAC sample and laser beams required for the experiment.

For measuring the pressure, the luminescence of highly calibrated materials is used. Very small ruby chips $(<10 \mu \mathrm{m})$ are usually placed inside the gasket aperture and away from the toroid for this purpose. Preferably, these chips should be in contact with one of the diamonds at the gasket aperture edge to prevent them from being heated too much. It is better to use $\mathrm{Sm}^{2+}: \mathrm{SrB}_{4} \mathrm{O}_{7}$ chips [10], since its emission has virtually no wavelength dependence with temperature.

A Nd:YLF laser (Quantronix Model 217D) capable of a maximum power of $55 \mathrm{~W}$ at $1053 \mathrm{~nm}$ in $\mathrm{TEM}_{01 *}$ mode is focussed onto the surface of the toroid. The $\mathrm{TEM}_{01 *}$ mode is 
used since the cross section profile of this mode can match the toroid shape fairly well. Our experience with nitrogen samples is that a very thin tungsten nitride film slowly forms on the toroid surface and protects the toroid when it is sufficiently heated. Although this process makes the toroid brittle, it renders it relatively inert and helps make the heating more uniform since the reflectivity of the toroid is slightly reduced. The heated toroid is still useful as a graybody for temperature measurements since the coating is relatively thin and transparent. In contrast, platinum toroids could only be heated for brief periods, probably due to the formation of platinum nitride that seemed to be highly reflective. Figure 2 demonstrates the effect of laser heating a tungsten toroid inside a pressurized DAC.

As long as the toroid aperture is sufficiently small, less than 25 microns, the volume within it should be at fairly uniform temperatures. It is this miniscule volume $(<10$ picoliters) that must be probed spectroscopically. It is vital that the toroid aperture is kept clear of anything but the sample.

To acquire vibrational spectra, Broadband Coherent Anti-Stokes Raman Spectroscopy (CARS) is employed. This is performed simultaneously with the laser heating described above and has a number of advantages over classical Raman Spectroscopy. The most important advantage is the ability to spatially, and even temporally, discriminate the signal from the Planck background.

For our broadband CARS experiments, we use a mode locked Q-switched Nd:YAG laser (plus necessary optics described below) to generate two pulses, one from a synchronously pumped broadband dye laser and another from frequency doubling the fundamental. A schematic of the system is shown in figure 3. The ML-QS Nd:YAG laser produces a train of about 60 to 80 pulses. Each pulse is $\sim 180 \mathrm{ps}$ long and there is $\sim 12 \mathrm{~ns}$ between pulses within the train. The pulse trains are set at a repetition rate of $\sim 1 \mathrm{KHz}$. The laser wavelength is $1064 \mathrm{~nm}$. One pulse from the train is selected and frequency doubled to provide the narrow band high frequency pulse $\left(\omega_{\mathrm{p}}\right)$. The other pulses in the train are also frequency doubled to $532 \mathrm{~nm}$ and used to synchronously pump a dye laser using the appropriate dye in methanol. For nitrogen, the dye chosen is Rhodamine 640. This laser has 
no tuning elements so that it lases broadband $\left(\sim 200 \mathrm{~cm}^{-1}\right.$ wide.) The dye concentration is varied so that the wavelength of the output is near $607 \mathrm{~nm}\left(\omega_{\mathrm{s}}.\right)$ The dye laser is intracavity dumped so that a single pulse about $30 \mathrm{ps}$ long has near megawatt peak power. These two pulses $\left(\omega_{\mathrm{p}}\right.$ and $\left.\omega_{\mathrm{s}}\right)$ must spatially and temporally overlap within the toroid hole. The CARS process produces a coherent emission at $\omega_{\mathrm{as}}=2 \omega_{\mathrm{p}}-\omega_{\mathrm{s}}$ that conserves photon momentum. There are both resonant $\left(\omega_{\mathrm{p}}-\omega_{\mathrm{s}}=\right.$ vibrational transition energy) and non-resonant contributions to the CARS process, the latter contributes to make the spectral peaks asymmetrical. [11] Because the experiment is done coherently and the sample is much larger than the wavelength of light used, the momenta of the photons are conserved and the anti-stokes pulse comes out in a known direction and can be easily spatially filtered. The Planck radiation, by contrast, comes out in all directions. This simple spatial filtering makes CARS much preferred over classical Raman spectroscopy for transparent laser heated samples.

\section{RESULTS}

Figure 4 represents typical data for a sample of nitrogen at $2000 \mathrm{~K}$, which was heated from an initial pressure of $10.3 \mathrm{GPa}$ at ambient temperature. The data in the figure has been corrected by dividing out the intensity, $\mathrm{I}\left(\omega_{\mathrm{s}}\right)$, of the dye laser at each wavelength from the raw data intensity. (i.e.: what the spectrum would look like if the dye laser output were spectrally flat.) The shoulder to the high energy side of the ground state peak is from relatively unheated nitrogen in front of and behind the toroid. Fortunately, the hot bands have very little contribution from the cooler nitrogen. The first and second excited states can be clearly seen, confirming that this sample is indeed hot within the toroid aperture. The data has been fit using equation 1 where subscript $\mathrm{j}$ is the vibrational level and $\omega_{\mathrm{p}}$ and $\omega_{\mathrm{s}}$ are the pump and stokes frequencies of the laser, respectively. The susceptibilities are represented as $\chi$ with the pk and nr superscripts being the third order peak and non-resonant 
susceptibility, respectively. $\Gamma$ is the HWHM linewidth. This equation has been simplified from reference 1 .

$I_{a s} \propto I_{s} \times\left[\left(\sum_{j} \frac{\Gamma_{j} \chi_{j}^{p k}\left(\omega_{j}-\omega_{p}+\omega_{s}\right)}{\left(\omega_{j}-\omega_{p}+\omega_{s}\right)^{2}+\Gamma_{j}^{2}}+\chi^{n r}\right)^{2}+\left(\sum_{j} \frac{\Gamma_{j}^{2} \chi_{j}^{p k}}{\left(\omega_{j}-\omega_{p}+\omega_{s}\right)^{2}+\Gamma_{j}^{2}}\right)^{2}\right]$

The fitting results in an error in pressure of approximately $0.7 \mathrm{GPa}$ and an error in temperature of about $200 \mathrm{~K}$, which are the same errors as one would get using the luminescence and graybody methods that are standard for DAC research. The high energy shoulder of the ground state peak, which is from relatively unheated material, can be used as a pressure sensor. Since the temperature dependence of the Raman shift of nitrogen is minimal below $900 \mathrm{~K}$, the pressure can be calculated from the peak position using data from reference 8 . The temperature can be calculated from the relative intensities of the excited state peaks since the material in the toroid aperture has a Boltzmann distribution. Therefore, the spectrum in figure 4 contains both pressure and temperature information and does not require additional spectra for the graybody emission and independent pressure sensor such as ruby or $\mathrm{Sm}^{2+}: \mathrm{SrB}_{4} \mathrm{O}_{7}$.

For this fit, the energies of $\omega_{\mathrm{j}}$ decreased by $\sim 28 \mathrm{~cm}^{-1}$ for the first excited level and $\sim 27.5$ $\mathrm{cm}^{-1}$ for the second. This is very close to the results obtained in reference 1 . If the potential becomes shallower (i.e.: the triple bond is breaking) due to increasing pressure, as suggested by shock experiments, [12] it can be directly observed by this method for samples at high pressure. 


\section{ACKNOWLEDGMENT}

We greatly appreciate the efforts of Ken Visbeck for his valued technical assistance and the advice of R. Boehler concerning the toroid fabrication technique. This work has been supported by the LDRD (00-ERD-024) and PDRP programs. This work was performed under the auspices of the U.S. Department of Energy by the University of California, Lawrence Livermore National Laboratory under contract No. W-7405-Eng-48.

Approved for public release; further dissemination unlimited 


\section{FIGURE CAPTIONS}

1: Configuration of the DAC diamonds, gasket and toroid. The typical path of the laser beams is also shown.

2: $\quad$ Upper panel: A sample of nitrogen at $10.5 \mathrm{GPa}$ and $295 \mathrm{~K}$ with a tungsten toroid held in place by the gasket.

Lower Panel: Same sample heated with $20 \mathrm{~W}$ of $1053 \mathrm{~nm}$ laser light. The pressure has increase by $\sim 2 \mathrm{GPa}$ and the temperature is $\sim 2000 \mathrm{~K}$. The toroid center is so bright that it falsely appears to have closed.

3: Schematic of CARS and laser heating setup. The dye laser wavelengths are typical for Rhodamine 640 in methanol. The movable delay line is used to synchronize the two laser pulses.

4: CARS spectrum of laser heated nitrogen. Asterisks represent the data corrected for $\mathrm{I}\left(\omega_{\mathrm{s}}\right)$. The data is fit using equation 1 and represented by the solid line using vibrational energies of 2352.8, 2324.8 and $2297.3 \mathrm{~cm}^{-1}$. The FWHM is $5.4 \mathrm{~cm}^{-1}$ for each peak and the $\chi^{\mathrm{pk}} / \chi^{\mathrm{nr}}$ ratios for the three peaks are 9.0, 3.6 and 1.1, respectively. 


\section{REFERENCES}

1. D. S. Moore, S. C. Schmidt, M. S. Shaw, and J. D. Johnson, J. Chem. Phys. 90, 1368 (1989).

2. S. C. Schmidt, D. S. Moore, M. S. Shaw, and J. D. Johnson, J. Chem. Phys. 91, 6765 (1989).

3. D. S. Moore, S. C. Schmidt, M. S. Shaw, and J. D. Johnson, J. Chem. Phys. 95, 5603 (1991).

4. S. C. Schmidt and D. S. Moore, Acc. Chem. Res. 25, 427 (1992).

5. S. C. Schmidt, D. S. Moore and M. S. Shaw, J. Chem. Phys. 107, 325 (1997).

6. S. C. Schmidt, D. S. Moore, M. S. Shaw, and J. D. Johnson, J. Chem. Phys. 98, 9379 (1993).

7. S. C. Schmidt, D. S. Moore and M. S. Shaw, J. Chem. Phys. 101, 3488 (1994).

8. A. S. Zinn, D. Schiferl, and M. F. Nicol, J. Chem. Phys. 87, 1267 (1987).

9. G. Zou, Y. Ma, H.-k. Mao, R. J. Hemley, and S. A. Gramsch, Rev. Sci. Instrum. 72, $1298(2001)$

10. Datchi, F., LeToullec, R., and Loubeyre, P., J. Appl. Phys. 81, 3333-3339 (1997).

11. J. W. Nibler and G. V. Knighten, in Raman spectroscopy (Springer-Verlag, West Germany, 1979) p253-299

12. Nellis, W. J., Holmes, N. C., Mitchell, A. C., and van Thiel, M., Phys. Rev. Lett. 53, 1661-1664 (1984). 


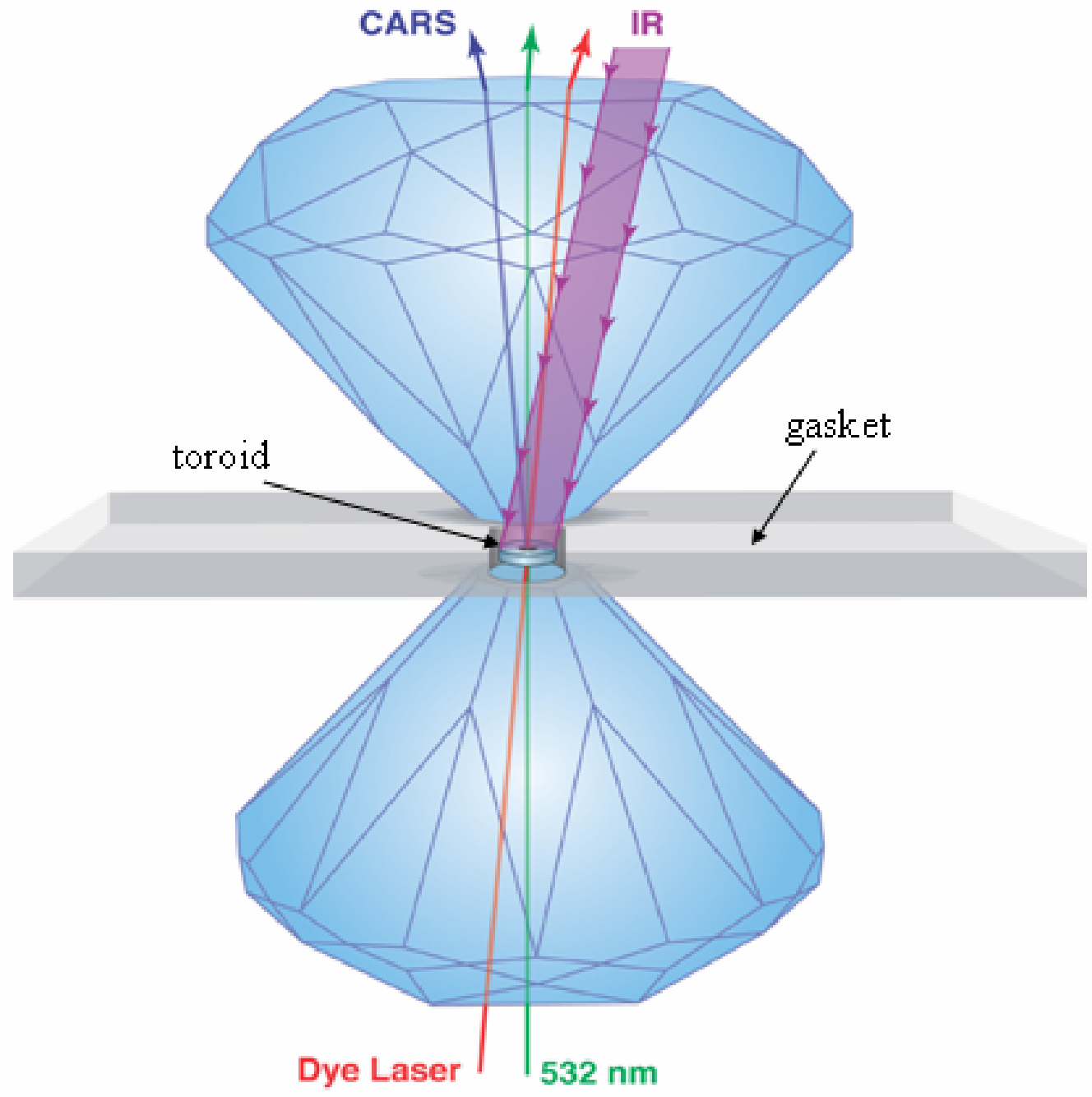

Approved for public release; further dissemination unlimited 

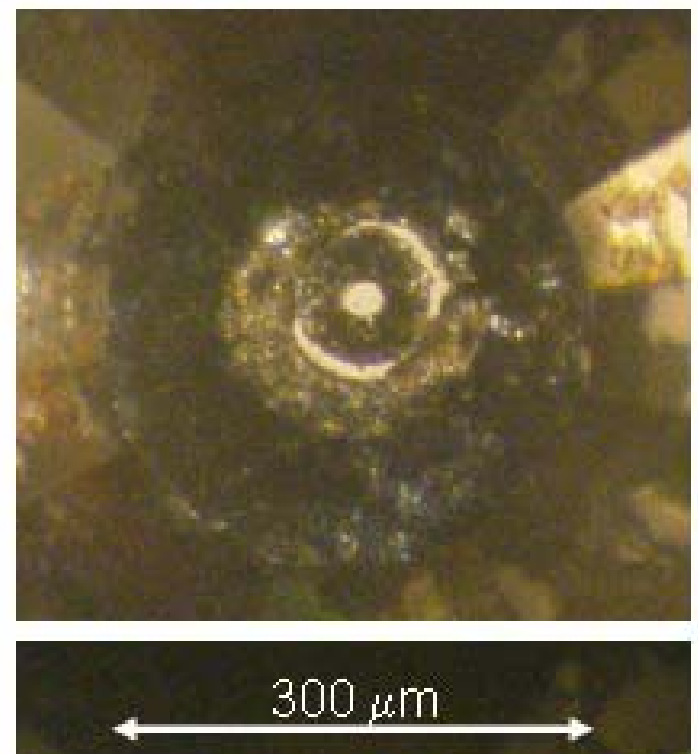

Approved for public release; further dissemination unlimited 


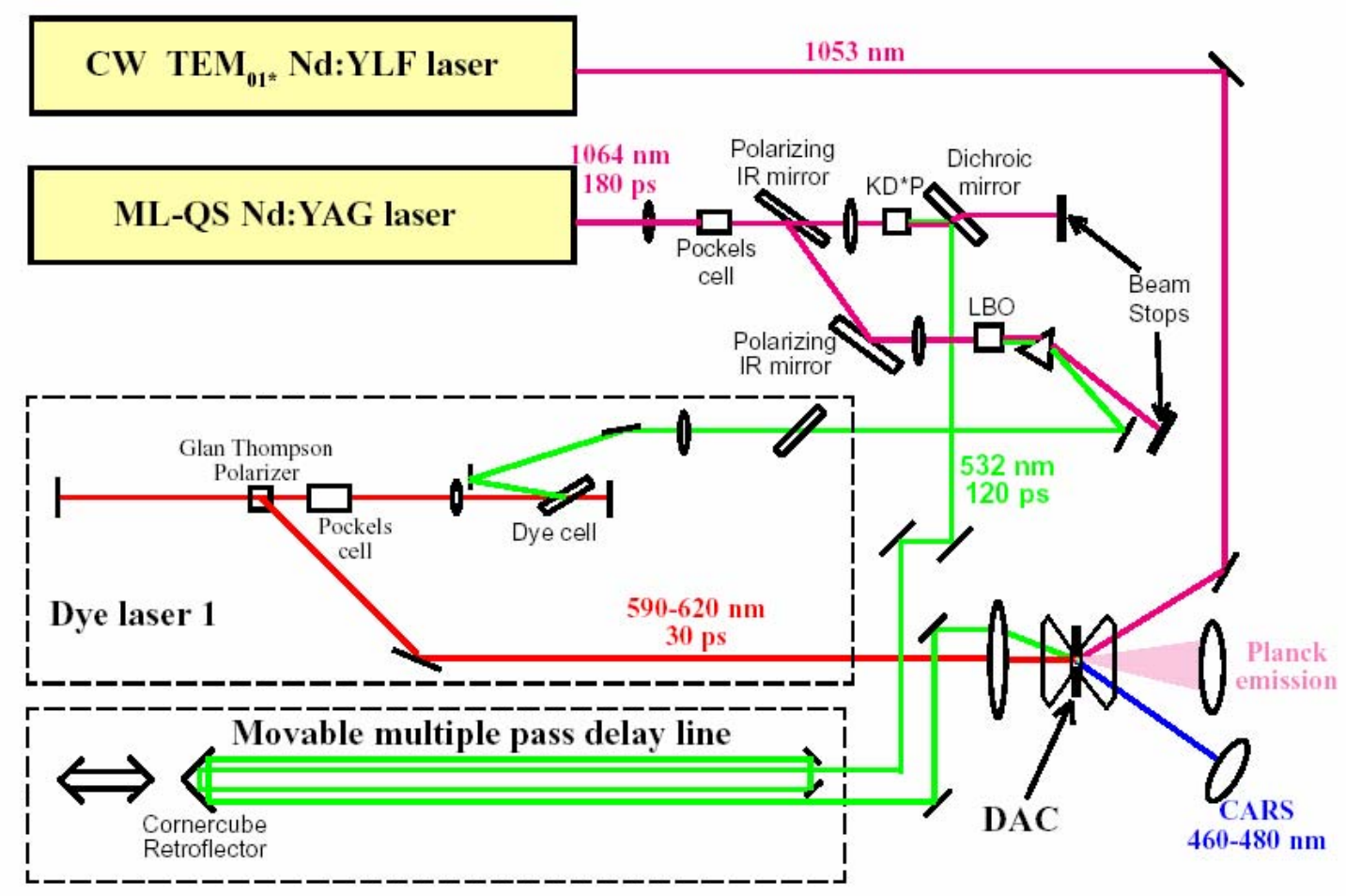

Approved for public release; further dissemination unlimited 


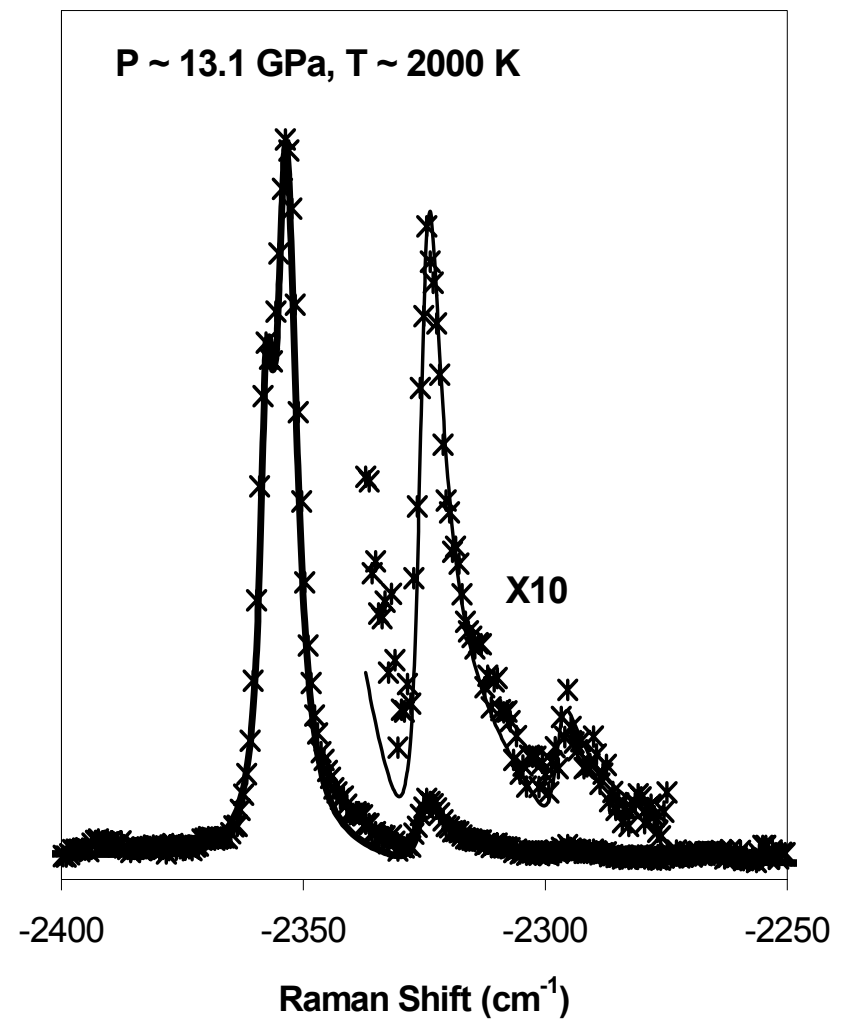

Approved for public release; further dissemination unlimited 